\title{
Using a 360-degree assessment of pediatric residency training: experience at Prince of Songkla University, Thailand
}

\author{
Somchit Jaruratanasirikul, Wassana Khotchasing \\ Department of Pediatrics, Faculty of Medicine, Prince of Songkla University, Songkla 90110, Thailand
}

Background: The Department of Pediatrics, Prince of Songkla University (PSU) with 7-10 pediatric residents per year has implied a 360-degree evaluating instrument for residency training since 2007.

Objective: We determined the competency ratings of pediatric residents during their training.

Methods: During 2007-2011, 23 pediatric residents finished the pediatric residency program. At each ward rotation, each pediatric resident was rated for competency skills by four different categories of raters: attending staff, nurses, medical students, and the patients' parents. The average score of each competency given by each category of raters was calculated, and was compared to scores of multiple-choice questions (MCQ) and constructed response questions (CRQ) of Thai Board of Pediatric Examination.

Results: The mean overall scores of each resident rated by the attending staff, nurses, medical students, and patients' parents increased with year of residency training. The mean overall scores of each resident rated by attending physicians were positively correlated with the MCQ $(r=0.42, p=0.04)$ and CRQ $(r=0.71, p<0.001)$ scores of the Thai Board of Pediatrics Examination.

Conclusion: The 360-degree assessments with ratings by attending physicians during the pediatric training are reliable for assessment the medical knowledge of the residents.

Keywords: Pediatric residency training, postgraduate training, 360-degree assessment, 360-degree evaluating instrument

Traditionally, competencies in professionalism, communication skills, and knowledge of the medical residents during the training have been evaluated by the attending physicians. However, these evaluations have some important limitations because the attending physicians have not had opportunity to observe the work of the residents during their learning period directly, nor their work relations with nurses, and patients. The 360-degree assessment, a program of assessment using multiple groups of raters who regularly interact with the trainees [1, 2], has been developed as a more reliable and valid tool for evaluation and feedback of resident physician competencies and performance from other sources other than attending physicians [3, 4].

In 2006, the Royal College of Pediatricians of Thailand endorsed the use of a 360-degree rating method to assess the competencies and performance

Correspondence to: Somchit Jaruratanasirikul, MD, Department of Pediatrics, Faculty of Medicine, Prince of Songkla University, Songkla 90110, Thailand.E-mail: somchit.j@psu.ac.th of Thai pediatric residents during their pediatric residency training [5]. The Department of Pediatrics of the Faculty of Medicine, Prince of Songkla University (PSU), with 7-9 pediatric residents per year implemented the 360-degree evaluating instrument for pediatric residency training in 2007. The residency training program is designed to ensure that its residents are developing the 6 core competencies: professionalism, communication skills, patient care/ clinical reasoning, procedural skills, medical knowledge, and leadership/teamwork. The program must have valid and reliable tools/instruments to assess the 6 competencies.

After 3 years of using the 360-degree rating method for pediatric residency training in PSU, we undertook this study to determine whether the competency rating of our residents during the training by faculty physicians differed from the nonfaculty raters (nurses, medical students, and patients' parents), and to determine whether these ratings were related to the scores of the Thai Board of Pediatric Examination achieved by the rated residents, which 
include both multiple choice question (MCQ) and constructed response question (CRQ) scores. To our knowledge, there have been no published studies assessing the use of the 360-degree evaluation for pediatric residency training or graduate medical education in Thailand.

\section{Materials and methods}

During our training program, the residents are assigned to attend as a house officer in the various pediatric wards for 9 months in the first year ( 6 months in the 4 general pediatric wards, 2 months in the nursery, and 1 month in the newborn intensive care unit), and 4-5 months in the second and third years (2-3 months in the general pediatric wards, 1 month each in the newborn and pediatric intensive care units).

During the period we were evaluating the 360degree program, at the end of each month of the ward rotations, each pediatric resident was rated for competencies by 4 different categories of raters, the attending physicians, nurses, medical students, and the patients' parents (Table 1). Six competencies were rated by attending physicians and 3 competencies by the nurses, medical students and patients' parents. Each competency was rated on a 9-point Likert scale (1-3 for unsatisfactory, 4-6 for satisfactory, and 7-9 outstanding).

This study was then a retrospective analysis of a prospective longitudinal study of the competencies of the pediatric residents in our institute. Twenty-three residents (22 females and 1 male) who finished the 3-year pediatric residency training during 2007-2012 from PSU were enrolled. Their mean age at the time of finishing the program was $28.8 \pm 1.2$ years. The average score of each competency given by each category of raters was calculated for each resident. The mean overall score by each group of raters was compared to the scores of the Thai Board of Pediatrics Examination achieved by the rated resident, which were based on both multiple choice questions (MCQs) and constructed response questions (CRQs).

\section{Statistical analysis}

Data were expressed as means and standard deviations. Chi square was used to compare the differences of the rating scores of each group of raters between the years of training. Pearson's correlation was used to identify correlations between the ratings of the competencies of the residents and the scores of the Thai Board of Pediatric Examination. Statistical differences were considered significant at a $p<0.05$. The protocol for this study was approved by the Institutional Review Board and the Ethics Committee of the Faculty of Medicine, Prince of Songkla University.

\section{Results}

The mean overall scores of each resident as rated by the attending physicians, nurses, medical students, and patients' parents increased with year of residency training, although they showed significant differences only in the ratings by the attending physicians and nurses (Table 2 and Figure 1). Among the four groups of raters, the scores of each competency rated by the medical students and patients' parents were near the highest scores for every resident, with no significant differences among the residents. The overall scores of each resident as rated by the attending physicians were positively correlated with the MCQ and CRQ scores of the Thai Board of Pediatric Examination (Figures 2 and 3), but were not correlated with the overall scores of the nurses, medical students, or patients' parents (Table 3).

Table 1. The four different groups of raters and the skills rated at each ward rotation

\begin{tabular}{|c|c|c|c|}
\hline Competency & Rater & No. of raters/month & No. of raters/year \\
\hline 1. Professionalism & Attending physicians & 2 & 12-18 \\
\hline 2. Communication skills & & & \\
\hline 3. Leadership/teamwork & & & \\
\hline 4. Patient care/clinical reasoning & & & \\
\hline 5. Procedural skills & & & \\
\hline 6. Medical knowledge & & & \\
\hline 1. Professionalism & Nurses & 3 & $20-30$ \\
\hline 2. Communication skills & Medical students & 3 & $20-30$ \\
\hline 3. Leadership/teamwork & Patients’ parents & 3 & $20-30$ \\
\hline
\end{tabular}


Table 2. The average scores (standard deviations) of resident competencies as rated by the 4 different groups of raters (full score is 9)

\begin{tabular}{llll}
\hline & Year 1 & Year 2 & Year3 \\
\hline Competencies rated by attending physicians & & & \\
1. Professionalism & $6.1(0.7)$ & $6.5(0.6)$ & $6.9(0.6)$ \\
2. Communication skills & $5.7(0.6)$ & $6.4(0.4)$ & $6.8(0.3)$ \\
3. Leadership/teamwork & $5.7(0.7)$ & $6.3(0.6)$ & $6.7(0.4)$ \\
4. Patient care/clinical reasoning & $5.5(0.6)$ & $6.1(0.5)$ & $6.5(0.5)$ \\
5. Procedural skills & $5.5(0.6)$ & $6.1(0.5)$ & $6.5(0.4)$ \\
6. Medical knowledge & $5.5(0.8)$ & $6.0(0.6)$ & $6.4(0.5)$ \\
Overall & $\mathbf{5 . 7 ( 0 . 7 )}$ & $\mathbf{6 . 2 ( 0 . 6 )}$ & $\mathbf{6 . 6 ( 0 . 5 )}$ \\
Competencies rated by nurses & & & \\
1. Professionalism & $7.2(0.4)$ & $7.5(0.4)$ & $7.7(0.3)$ \\
2. Communication skills & $7.1(0.3)$ & $7.4(0.3)$ & $7.7(0.3)$ \\
3. Leadership/teamwork & $6.9(0.3)$ & $7.3(0.3)$ & $7.6(0.4)$ \\
Overall & $\mathbf{7 . 1 ( 0 . 3 )}$ & $7.4(\mathbf{0 . 3})$ & $7.7(\mathbf{0 . 3})$ \\
Competencies rated by medical students & & & \\
1. Professionalism & $8.2(0.2)$ & $8.5(0.2)$ & $8.7(0.1)$ \\
2. Communication skills & $8.1(0.1)$ & $8.5(0.1)$ & $8.7(0.1)$ \\
3. Leadership/teamwork & $8.0(0.2)$ & $8.3(0.2)$ & $8.7(0.1)$ \\
Overall & $\mathbf{8 . 1 ( 0 . 2 )}$ & $\mathbf{8 . 4 ( 0 . 2 )}$ & $\mathbf{8 . 7 ( 0 . 1 )}$ \\
Competencies rated by patients' parents & & & \\
1. Professionalism & $7.9(0.2)$ & $8.0(0.2)$ & $8.2(0.2)$ \\
2. Communication skills & $7.8(0.2)$ & $7.9(0.3)$ & $8.2(0.2)$ \\
3. Leadership/teamwork & $7.8(0.2)$ & $7.9(0.3)$ & $8.2(0.2)$ \\
Overall & $\mathbf{7 . 8 ( 0 . 2 )}$ & $\mathbf{7 . 9 ( 0 . 3 )}$ & $\mathbf{8 . 2 ( 0 . 2 )}$
\end{tabular}

\section{Likert scores}

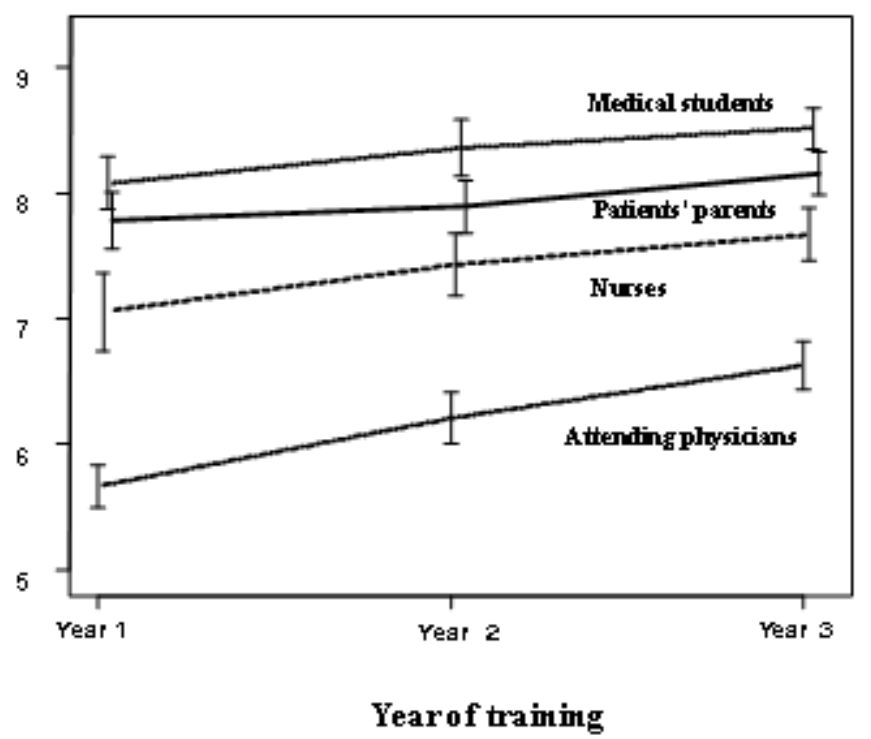

Figure 1. The mean overall scores of the residents' competencies rated by attending physicians, nurses, and patient's parents increased with year of residency training 


\section{MCQ scores (\%)}

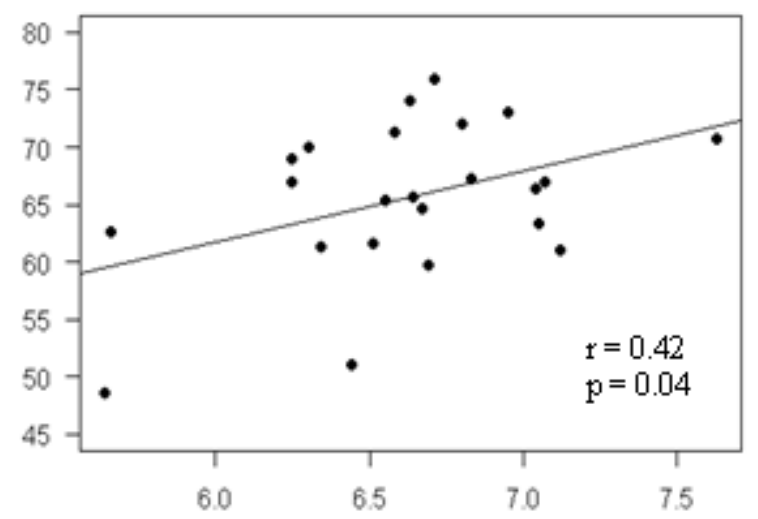

Overall competency scores rated by attending p lysicians

Figure 2. The correlation of the mean overall scores of resident as rated by the attending physicians and the multiple choice question (MCQ) scores of the Thai Board of Pediatric Examination

\section{CRQ scores (\%)}

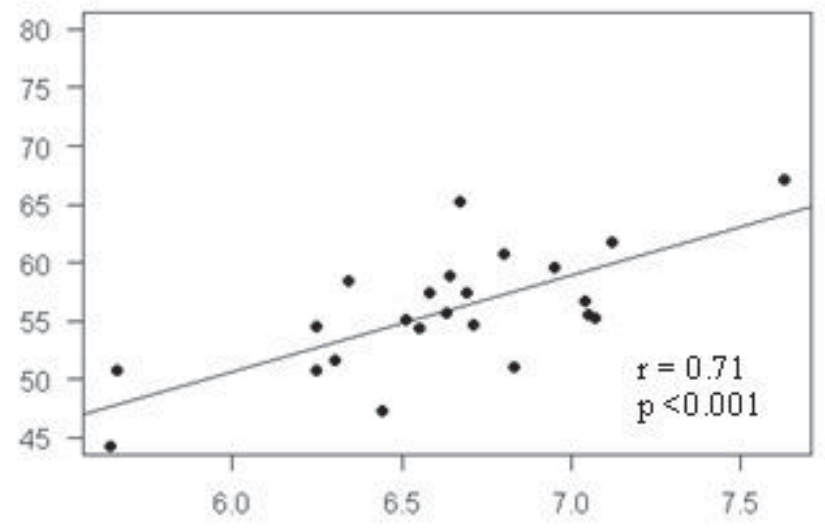

Overall comp etency sco res rated by attending physicians

Figure 3. The correlation of the mean overall scores of residents as rated by the attending physicians and the constructed response question (CRQ) scores of the Thai Board of Pediatric Examination

Table 3. Correlation of overall competency scores of residents rated by the four different groups of raters and MCQ and CRQ scores of the Thai Board of Pediatric Examination

\begin{tabular}{lllll}
\hline Groups of raters & \multicolumn{2}{r}{ MCQ } & \multicolumn{2}{c}{ CRQ } \\
\cline { 2 - 5 } & r & $\boldsymbol{p}$ & r & $\boldsymbol{p}$ \\
\hline Attending physicians & 0.42 & 0.04 & 0.71 & $<0.001$ \\
Nurses & 0.22 & 0.31 & 0.16 & 0.47 \\
Medical students & -0.15 & 0.52 & 0.06 & 0.81 \\
Patients' parents & -0.14 & 0.53 & 0.53 & 0.81
\end{tabular}




\section{Discussion}

Our study compared the assessments of the residents by four different groups of raters who worked or interacted with our pediatric residents as a continuous 360-degree evaluation during the 3-year training program in our institute, and then compared these evaluations with those of the Thai Board of Pediatric Examination. All the residents were rated with a very high score by the medical students and the patients' parents in all competencies through all three years of training. This outcome was differed partly from the outcome of a study by Chandler et al. in which the patients and their families scored the resident competencies significantly lower than the physicians and nurses [2]. The higher scores in our study could be explained by noting that medical students in Thai culture have a closer relationship with the residents they learn from than seems to be the case in most western settings, and they spend considerable time together as a team taking care of their patients in the wards and also during on call duties, and thus may either have a closer opportunity to observe the residents' work, and/or be somewhat disinclined to criticize them officially. For the high rating scores by the patients' parents, there are several possible explanations: first, it is the Thai culture that patients give high respect to their physicians for the medical care of their children; second, the parents questioned during the study were highly satisfied with the competencies of the residents; and third, our residents did actually communicate well and display high professionalism. The competency scores rated by the nurses were also in the high ranges, but were significantly lower than the ratings by the medical students and the patients' parents. This outcome was similar to the outcome from study by Brinkman et al. in which the nurses scored the residents' competencies significantly lower than the patients' parents [6]. The explanation for the differences in our study is that the nurses evaluated the residents and compared the competencies in professionalism and communication skills with the attending physicians, and thus would be less likely to give scores as high as the medical students and patients' parents, who were less familiar with the work of senior physicians. Among the four groups of raters, the scores of the attending physicians were significantly lower than the other raters, because the attending physicians evaluated the actual competencies of the residents in their knowledge, clinical reasoning, procedural skills, and communication skills, whilst the medical students and parents, in particular, would have their scores influenced by positive personal interactions with the residents as they treated the patients. As the residents gained more experience, in their second and third years, the competency scores given by the attending physicians significantly increased.

Knowledge competency can be formally evaluated by examination. The results of our study showed that the medical knowledge competency scores and the overall competency scores of the residents in their third year of training were significantly positively correlated with both the MCQ and CRQ scores of the Thai Board of Pediatric Examination. This positive correlation indicates that the evaluations of resident competency by the attending physicians were reliable and valid, particularly concerning knowledge competency. The correlation is more clearly evident between overall competency and the CRQ scores than with the MCQ scores, which can be explained by noting that with the CRQ the residents have to express their knowledge in a written short essay, but with the MCQ they simply choose one of the 5 items in the MCQ test, meaning that they can guess at answers, even if they did not know the answer, with a written answer they must know the answer.

The use of 360-degree assessment has been advocated as a mean of gaining additional feedback on resident performances from sources other than attending physicians [7, 8]. The purpose of the 360degree evaluation is to enhance the evaluation process and provide additional information for feedback and teaching. In our study, the positive evaluations by the medical students, patients' parents, and the nurses reflected their positive attitude towards the competencies of our residents, which would in turn make our residents feel positive with the assessments and work harder to improve their competencies in their career work.

We did not include the residents' self-evaluation in our study because these represented only one data set from one person performed once yearly. Moreover, previous studies have shown disagreements between self-evaluations and assessments by external raters in both inflated self-assessments $[9,10]$ and deflated self-assessments $[4,11]$, indicating that it is very difficult for a resident to give an accurate evaluation on their own work or performance.

Our study is the first report concerning the use 
of a 360-degree evaluation to assess resident competencies in a postgraduate training institute in Thailand. The success of the use of the 360-degree evaluation tool in our institution required the understanding and cooperation of the medical care team to complete the evaluations regularly, and the participation of our supporting medical education team to distribute the evaluation sheets to and collect them back from the many raters.

The study has some limitations. First, the number of residents being evaluated was relatively small. Second, it was undertaken during the first three years of implementation of the 360-degree program, and thus was forced to contend with whatever learning curve problems that could potentially cause problems. We do not think that this is a difficult program to implement, and such problems were not really in any way significant. Despite the short period, we saw a positive impact and improvement in knowledge, procedural skills, communication skills, and leadership/ teamwork of our resident competencies, as it was, and is, a longitudinal evaluation and feedback process from the first year through the third year of training. Finally, the overall scores of competencies correlated well with the final scores of the Thai Board of Pediatric Examination.

In conclusion, our study suggests that the 360degree instrument, with ratings by attending physicians, nurses, medical students, and patients' parents, can be practically used to evaluate the competencies of residents during their 3-year residency training. Such comprehensive assessment can also be useful in providing feedback for the residents concerning their professionalism, medical knowledge, procedural skills, communication skills, and leadership/teamwork. The overall scores rated by the attending physicians during the third year of training were reliable assessments of the medical knowledge of the residents, and were positively correlated with the MCQ and CRQ scores of the Thai Board of Pediatric Examination.

The authors have no conflict of interest to declare.

\section{References}

1. Meng L, Metro DG, Patel RM. Evaluating professionalism and interpersonal and communication skill: implementing a 360-degree evaluation instrument in an anesthesiology residency program. J Grad Med Educ. 2009; 1:216-20.

2. Chandler N, Henderson G, Park B, Byerley J, Brown WD, Steiner MJ. Use of a 360-degree evaluation in the outpatient setting: the usefulnesss of nurse, faculty, patient/family, and resident self-evaluation. J Grad Med Educ. 2010; 2:430-4.

3. Brett JF, Atwater LE. 360-degree feedback: accuracy, reaction and perceptions of usefulness. J Appl Psychol. 2001; 86:930-42.

4. Joshi R, Ling FW, Jaeger J. Assessment of a 360-degree instrument to evaluate residents' competency in interpersonal and communication skills. Acad Med. 2004; 79:458-63.

5. The Royal College of Pediatricians of Thailand. Curriculum for pediatric residency training [online] 2012. [cited 1 September 2012] Available from: www. thaipediatrics.org/attchfile/course05.pdf

6. Brinkman WB, Geraghty SR, Lanphear BP, Khoury JC, Gonzalez del Rey JA, DeWitt TG, et al. Evaluation of residents communication skills and professionalism: a matter of perspective? Pediatrics. 2006; 118:1371-9.

7. Dubinsky I, Jennings K, Greengarten M, Brans A. 360-Degree physician performance assessment. Healthc Q. 2010; 13:71-6.

8. Overeem K, Wollersheim H, Driessen E, Lombarts K, van de Ven G, Grol R, et al. Doctors' perceptions of why 360-degree feedback does (not) work: a quality study. Med Educ. 2009; 43:874-82.

9. Kruger J, Dunning D. Unskilled and unaware of it: how difficulties in recognizing one's own incompetence lead to inflated self-assessments. J Pers Soc Psychol. 1999; 77:1121-34.

10. Tousignant M, DesMarchais JE. Accuracy of student self-assessment ability compared to their own performance in a problem-based learning medical program. A correlation study. Adv Health Sci Educ Theory Pract. 2002; 7:19-27.

11. Lipsett PA, Harris I, Downing S. Resident self-other assessor agreement: influence of assessor, competency, and performance level. Arch Surg. 2011; 146:901-6. 